D.O.I.: $10.3895 / \mathrm{S} 1808-04482010000200003$

\title{
DEFINIÇÃO DE PARÂMETROS PARA A CONSTRUÇÃO DE MODELOS DE PLANEJAMENTO AGRÍCOLA: UM CENÁRIO PARA OTIMIZAÇÃO DE PROCESSOS AGROINDUSTRIAIS
}

\section{DEFINITION OF PARAMETERS FOR THE CONSTRUCTION OF MODELS OF AGRICULTURAL PLANNING: A SCENARIO FOR OPTIMIZATION OF AGROINDUSTRIAL PROCESS}

\author{
Carlos Alessandro Neiverth Oliszeski ${ }^{1}$; João Carlos Colmenero ${ }^{2}$ \\ ${ }^{1}$ Universidade Tecnológica Federal do Paraná - UTFPR - Ponta Grossa - Brasil \\ carlosoliszeski@uol.com.br \\ ${ }^{2}$ Universidade Tecnológica Federal do Paraná - UTFPR - Ponta Grossa - Brasil \\ colmenero@utfpr.edu.br
}

\begin{abstract}
Resumo
Os setores agroindustriais vêm cada vez mais aprimorando o conhecimento tecnológico dentro de suas áreas de atuação, não somente pela concorrência, que é cada vez mais acirrada, mas também pela própria e simples sobrevivência no mercado. Isso faz com que haja uma necessidade de se conseguir uma otimização de ações, que no caso da agroindústria é traduzido como produzir e cultivar alimentos com economia de investimentos, evitando o desperdício de matéria-prima, insumos e gastos desnecessários, ainda mais, pelo fato de ser um setor que conta com as adversidades do clima. A pesquisa operacional, como ciência, estrutura processos, propondo um conjunto de alternativas de ação, fazendo a previsão e a comparação de valores, de eficiência e de custos. Já os modelos de otimização, são utilizados quando existe o interesse em se encontrar a alternativa que melhor atenda a um ou simultaneamente a vários objetivos, dado um conjunto de restrições, geralmente lineares. Tendo como princípio a obtenção de soluções ótimas para os processos agroindustriais de planejamento e escolha de culturas agrícolas, o objetivo do presente trabalho foi determinar os parâmetros a serem utilizados para a construção de eventuais modelos de otimização e que devem ser considerados para que haja a maximização de lucros em propriedades agrícolas, com melhor aproveitamento de terras, capital e mão-de-obra, nos sistemas de produção propostos, bem como a minimização dos riscos de se optar por determinadas culturas.
\end{abstract}

Palavras-chave: otimização, agroindústria, pesquisa operacional, culturas agrícolas.

\section{Introdução}

Os setores da agroindústria sejam eles, de processamento, insumos, distribuição e produção primária vêm como em outros setores, cada vez mais aprimorando o conhecimento tecnológico dentro de suas áreas de atuação, não somente pela concorrência, que é cada vez mais acirrada, mas também pela simples sobrevivência no mercado. Seja por questões de gerenciamento, controladoria 
ou tomadas de decisão, a evidência da empresa do futuro deverá ser baseada no equilíbrio entre a gestão, o controle de seus projetos e a constante busca por tecnologias e o desenvolvimento de novos produtos.

Tomar decisões exige um embasamento muito forte, principalmente quando estão envolvidos grandes investimentos. Caso contrário, corre-se o risco de se realizar investimentos de forma indevida, ou deixar de fazê-los quando eram necessários.

Paralelamente a essa situação estão os pequenos produtores, que geralmente possuem uma baixa renda e retiram seu sustento do pequeno espaço de terra que dispõem em sua propriedade. Essa classe normalmente não possui base científica ou conhecimento necessário para planejar adequadamente o seu processo produtivo e dele retirar os melhores resultados possíveis, mesmo com o auxílio de um profissional.

Por diversas vezes, as pessoas ligadas ao ramo da agroindústria não conseguem selecionar as melhores culturas a serem cultivadas em seus solos, não atentam para os melhores resultados de produtividade com relação ao espaço disponível ou simplesmente, deixam de lado a possibilidade de obter maior lucro com a mesma quantidade de recursos disponíveis.

Mas, tomar boas decisões raramente é uma tarefa fácil. Os problemas enfrentados pelos tomadores de decisão no ambiente comercial competitivo e de ritmo frenético de hoje em dia são geralmente de extrema complexidade e podem ser resolvidos por vários cursos de ação possíveis. A avaliação dessas alternativas e a escolha do melhor curso de ação representam a essência da análise de decisão.

A utilização de técnicas de otimização fundamentadas em processos que envolvem múltiplos objetivos vem crescendo de forma significativa em diversas áreas, especialmente no que se refere a problemas de tomada de decisão no setor agrícola.

Partindo desse princípio, o desenvolvimento de modelos que contribuam para a compreensão mais eficaz dos problemas enfrentados por propriedades rurais, e que, proponham sistemas e métodos que dêem suporte às atividades da agroindústria, seria de grande auxílio para prover um melhor planejamento dos processos.

Assim, a construção de modelos matemáticos como ferramentas de pesquisa e promotora de eficiência decisória pode ser um passo importante para atingir, com maior exatidão, os resultados de qualquer estratégia adotada, bem como prever e controlar possíveis falhas durante sua execução.

\section{O planejamento no empreendimento rural}

Segundo Vilckas (2004), a elaboração e implementação do planejamento no setor rural representam um desafio muito grande, tendo em vista que os empreendimentos desse setor estão 
sujeitos a um grande numero de variáveis, como a dependência de recursos naturais, a sazonalidade de mercado, a perecibilidade dos produtos, o ciclo biológico de vegetais e de animais eo tempo de maturação dos produtos.

Outro fator importante é a possibilidade mudanças imediatas na produção, pois uma vez realizado o investimento, é necessário aguardar o resultado da produção e escoá-la rapidamente, mesmo em condições desfavoráveis de mercado, a não ser que o produto possa ser estocado à espera de melhores condições de venda. Essas particularidades resultam em uma maior complexidade no gerenciamento do empreendimento rural, que pode ser reduzida com o emprego de técnicas gerenciais que garantam sua competitividade a longo prazo.

Segundo a Confederacao da Agricultura e Pecuária do Brasil a utilização de ferramentas gerenciais pelos produtores ainda é reduzida, mas eles têm percebido que apenas conhecimentos técnicos de produção/criação, embora fundamentais, não bastam, e estão reconhecendo a importância da administração, em especial a do planejamento, em suas atividades.

Os benefícios da administração para o desempenho econômico das propriedades rurais são muitos e significativos. Empreendimentos que tem suas atividades planejadas obtêm melhores resultados na gestão de seus negócios. Entre os vários fatores que contribuem para o sucesso de um empreendimento rural, muito se deve à habilidade gerencial do seu proprietário, que deve ser entendida não somente como a dedicação às tarefas rotineiras e à relação com seus funcionários, mas envolve também uma visão sistêmica do processo produtivo.

\title{
2.1 O que produzir?
}

Em qualquer empreendimento rural, a primeira questão que se coloca é qual ou quais serão os produtos a serem produzidos e em quais quantidades, tendo em vista os recursos disponíveis e o retorno desejado. Participam dessa decisão diversos fatores que podem ser alinhados segundo suas principais vertentes: características dos recursos disponíveis e condições de mercado.

\begin{abstract}
A primeira vista, o espectro de alternativas possíveis, na escolha do elenco de produtos pelo qual se pode optar, parece bastante amplo. Entretanto, esse elenco se restringe à medida que se analisem as características dos recursos disponíveis. Assim, o primeiro estágio de análise supõe que se faça uma avaliação do potencial natural, sem o uso do que se poderia denominar como adequadores de produtividade muito complexos ou caros, tais como corretores de solo, equipamentos ou construções sofisticadas. (CAIXETA-FILHO, 2004).
\end{abstract}

\subsection{Quanto produzir?}

É evidente que quando se fala em empresa rural a quantidade a produzir estará inicial e fortemente vinculada a decisão do que produzir (mínimo economicamente aceitável), a área 
disponível (máximo possível) e a demanda ou restrições do mercado, isto é, quantidade recomendável ou contratada.

Se o máximo recomendável ou contratado for compatível com o máximo possível, tanto melhor, ocupa-se toda a área disponível. Deve-se observar que o máximo disponível também estará condicionado às necessidades de recuperação do solo ou cobertura de áreas degradadas pelo uso, exigindo uma rotatividade dos espaços de produção.

Dependendo do produto da empresa rural e da capacidade econômica do produtor, deve-se considerar também a hipótese de estocar o produto em face de condições melhores de venda futura. Desta forma, restrições de mercado podem ser atenuadas.

A diversidade produtiva tem por vantagens a economia de escopo, consorciada ou intercalada (produção com menores custos), redução do risco (eventos que atinjam um produto não atingem necessariamente os outros), uso mais balanceado dos recursos produtivos ou ainda alguma auto-suficiência para a empresa rural. Entretanto, essas alternativas podem introduzir complexidade administrativa, dispersão de esforços e em algumas situações concorrência pelos recursos produtivos (equipamentos e mão-de-obra). Alguma diversidade é sempre positiva, tendo em vista a redução de riscos.

Quando dedicados a produtos únicos, estes devem ser tais que ocupem nichos específicos de mercado ou tenham demandas contratadas com exclusividade.

\subsection{Para quando produzir?}

Como para todos os demais produtos, houve época em que a demanda de produtos primários era superior a oferta e produzia-se tanto quanto fosse possível, pois se tinha a certeza de que comercializar era só uma questão de ter os meios para transportar e atingir os mercados. Assim, "quando entregar" não era uma questão tão relevante.

Havia demanda para receber todos os produtos primários ou artesanalmente manipulados e estocar era uma preocupação do cliente ou usuário. Entretanto, ao longo do tempo, em face da grande oferta, modernização dos meios de transporte e exigências do mercado consumidor, uma gama significativa de produtos primários passou a fazer parte de cadeias agroindustriais tornando-se insumo para enorme diversidade de produtos industrial. (CAIXETA-FILHO, 2004).

Estocar deixou de ser uma preocupação do cliente ou usuário para ser uma preocupação da indústria transformadora e do comércio. Estes segmentos, entretanto, muito mais atentos as economias de escala, passaram a pressionar as empresas rurais a fornecer seus produtos com a frequência requerida pelo processamento industrial, evitando-se ao máximo estoque e perdas desnecessárias. Assim, passou-se a exigir entregas de matérias-primas mais constantes em prazos determinados. 
As empresas rurais vinculadas as cadeias agroindustriais tem, portanto, datas ou períodos de entrega bem determinados, mesmo considerando os significativos riscos das variações climáticas existentes. Isso significa que consideráveis esforços têm que ser feitos para controlar os desvios climáticos, quando possível. Tome-se como exemplo o bombardeio para fragmentação de granizo em plantações de maçã, ou a proteção das culturas e criações como no caso do uso de ventiladores para manutenção de temperatura em granjas, e a plasticultura no plantio de morangos e verduras. Além desses esforços relativos a redução dos riscos climáticos, deve-se dispor de tácnicas de gestão que proporcionem meios de executar cada etapa do processo de produção de forma controlada, ou mesmo dispor da matéria-prima no momento desejado pela manipulação de diferentes mudas ou sementes com diferentes períodos de plantio e maturação, no caso de culturas (CAIXETA-FILHO, 2004).

\section{Administração de propriedades e atividades rurais}

Segundo Lima et al (2001), o desenvolvimento teórico e prático da administração rural toma por base duas abordagens: da economia rural, com ênfase no uso mais eficiente de recursos, ou seja, está relacionada a atividades de análise econômica; e da ciência administrativa, com destaque para a análise dos aspectos inerentes à empresa rural e suas inter-relações com o meio ambiente, contemplado os diferentes elementos do processo administrativo.

Conforme Brossier (1990), a tomada de decisão e os modos de gestão nas empresas levam os administradores a uma adaptação do seu comportamento no momento em que buscam uma solução satisfatória ou ótima para a situação ou problema. Pode-se inferir que não é o indivíduo sozinho que toma as decisões e muitas vezes o critério de decisão não é a otimização, mas a obtenção de uma solução satisfatória ou aceitável, ou seja, a empresa tem relações com o ambiente e isso lhe garante benefícios e lhe impõe limitações.

\subsection{A contabilidade de custos nas empresas rurais}

Segundo Callado (2009), facilmente, pode-se constatar que, embora não seja verdade absoluta, a administração das empresas ligadas ao agronegócio brasileiro ainda se desenvolve dentro de critérios tradicionais que apresentam um padrão de desempenho restrito, considerando seu potencial global.

A contabilidade rural e uma ferramenta gerencial pouco utilizada pelos produtores brasileiros, pois e vista como uma técnica complexa em sua execução e que apresenta um baixo retorno pratico.

Sobre a importância de um sistema de custos, Marion (2000), destaca seus objetivos dentro da empresa afirmando que refletem sua importância como ferramenta básica para a administração de qualquer empreendimento, especialmente na agropecuária,cujos espaços de tempo entre produção e vendas, ou seja, entre custos e receitas, fogem a simplicidade de outros tipos de negócios. 
A classificação proposta por Marion (2000) contempla as principais expectativas conceituais sobre custos rurais por sua adequação, referencias e enfoque, apresentando três tipos:

a) Quanto a Natureza: Classificação que se refere à identidade daquilo que foi consumido na produção.

b) Quanto à identificação com o Produto: Classificação que se refere a maior ou menor facilidade de identificar os custos com os produtos, através de uma medição precisa dos insumos utilizados, da relevância do seu valor ou da apropriação dos gastos por rateio.

c) Quanto a sua Variação Quantitativa: Classificação que se refere ao fato de os custos permanecerem inalterados ou variarem em relação às quantidades produzidas. Ou seja, os custos podem variar proporcionalmente ao volume produzido ou podem permanecer constantes, independentemente do volume.

\subsection{Representação quantitativa dos custos rurais}

Os custos de fabricação encontrados dentro das atividades produtivas de uma organização agroindustrial podem ser classificados entre custos diretos e indiretos.

Callado et al (2001), apresentam um modelo para estruturar quantitativamente os custos globais e parciais de produção rural para uma empresa agroindustrial. Para identificar o valor das margens de contribuição para cada um dos produtos devemos encontrar seus respectivos custos unitários.

O planejamento das operações, demandado pela crescente competitividade sugere que uma perspectiva analítica sobre o comportamento dos custos de fabricação deve ser desenvolvida e aperfeiçoada. Mais importante se torna o processo de apuração e alocação dos custos indiretos de fabricação.

Para visualizar a perspectiva que a apuração dos custos globais de uma determinada empresa rural oferece, observa-se a estrutura da equação 1.

$$
\mathrm{Cg}=\sum_{i=1}^{n} \mathrm{~V} i \mathrm{P} i++\sum_{k=1}^{n} \mathrm{~A} k \mathrm{P} k+\sum_{m=1}^{n} \mathrm{Im} \mathrm{P} m+\sum_{q=1}^{n} \mathrm{C} q \mathrm{P} q
$$

Onde

$\mathrm{Cg}-$ Custos Globais

$\mathrm{V}$ - Elemento de custo das atividades vegetais;

A - Elemento de custo das atividades animais;

I - Elemento de custo das atividades agroindustriais;

C - Elemento de custo das atividades complementares; 
P - Custo unitário do item de custo;

$i$ - Quantidade de elementos de custos das atividades vegetais;

$k$-Quantidade de elementos de custos das atividades animais;

$m$ - Quantidade de elementos de custos das atividades agroindustriais

$q$ - Quantidade de elementos de custos das atividades complementares.

\section{A tomada de decisão}

Conforme Brossier (1990) são antigos os esforços no sentido de modelar o processo de tomada de decisão. A teoria microeconômica da firma constitui o primeiro esforço de elaboração de um modelo que foca o comportamento dos produtores (o quê, quanto, por que e para quem produzir). Seu objetivo não é compreender o funcionamento das unidades e sim conhecer como funciona uma economia global que age sobre pequenas unidades. Com esse modelo é possível determinar quais são os produtos que serão produzidos (o que), em que nível (quanto) e com quais fatores de produção (como). Assim a decisão de produzir será tomada quando os custos de uma unidade suplementar são inferiores às receitas, ou seja, quando for possível obter lucro com a atividade.

Os agricultores gerenciam fatores e técnicas para produzir bens e serviços. Assim, eles tomam decisões técnicas e econômicas, baseados em regras e princípios escolhidos rapidamente. Essas decisões, contudo, não estão livre da influencia de fatores que muitas vezes não são conhecidos a priori. Ou se são, é a usa possibilidade de ocorrência que é desconhecida.

Pindyck e Rubinfeld (1994) dizem que é preciso escolher o grau de risco que que se está disposto a assumir. Ou seja, no momento em que as condições futuras são incertas, tomar decisão envolve riscos. Assim, a escolha do grau de risco a ser assumido dependerá de alguns fatores pelos quais os administradores poderão ser influenciados. Esses fatores podem ser a busca de maiores rendimentos, melhores e maiores oportunidades, probabilidade de ocorrência, etc. Assim, a diversificação das atividades apresenta-se como uma possibilidade, desde que ela aumente a capacidade da empresa de maximizar a utilização dos recursos. Da mesma forma temos a tomada de decisão quanto a vender ou não o resultado da produção em um determinado período ou época do ano. No caso dos produtores rurais, a falta de informações sobre o comportamento do mercado e sua composição de custos de produção, os deixa à mercê de posições especulativas, as quais multiplicam em muito os riscos e incertezas de suas atividades.

O produtor rural faz parte, hoje, de um amplo complexo de relações econômicas e contratuais, que estão ligadas em cadeia, dentro de um contexto conhecido como agronegócio. O 
tamanho de sua atividade não é mais medido pela área física que possui ou ocupa, mas pela renda que o produtor consegue extrair de cada hectare dessa área ou de cada animal que produz.

\subsection{Tomada de decisão multicritério}

O Método de Programação Matemática sob Múltiplos Critérios consiste na otimização de várias funções-objetivo, como, por exemplo, maximizar margem bruta e nível de emprego e minimizar risco econômico, sujeito a um conjunto de restrições também lineares, como disponibilidade de terra, horas de trator por período e disponibilidade de água.

No entanto, a ênfase em diferentes objetivos poderá afetar a escolha da técnica, o caminho para o desenvolvimento e as prioridades da pesquisa. Devem-se considerar, para um planejamento consistente, todos os objetivos impostos sobre a área estudada.

Conforme (Gomes et.al, 2002) nos problemas multicritério, é bastante comum que, para o agente de decisão, alguns critérios sejam mais relevantes do que outros. Por motivos diversos, entre os quais estão suas preferências pessoais (razoavelmente explicitadas ou completamente subjetivas), o agente de decisão pode considerar alguns critérios menosjuntamente ou mais importantes do que os demais. As medidas que expressam a importância relativa entre os critérios são denominadas pesos dos critérios. O conjunto de pesos atribuídos aos diferentes critérios denomina-se vetor de pesos. A matriz de decisão, juntamente com o vetor de peso, constitui toda a informação necessária, em princípio, para a resolução dos problemas multicritério discretos.

Como os pesos têm papel fundamental na resultante do conflito entre critérios, estes influenciam de maneira decisiva os resultados obtidos. Assim, a atribuição de valores aos pesos dos critérios deve ser bastante cuidadosa, tendo em vista, sobretudo, o caráter subjetivo desta tarefa. Afinal, os pesos devem refletir, o mais fielmente possível, as preferências do agente de decisão.

Existem diversas técnicas para atribuição de pesos aos critérios, algumas diretas e outras envolvendo métodos mais sofisticados. Como exemplo de técnicas diretas, podem ser citadas:

a) Ordenação simples - o agente de decisão deve priorizar os critérios na ordem de sua preferência. Ao critério menos importante associa-se o valor 1, ao penúltimo, o valor 2, e assim sucessivamente. Ao critério mais importante deve-se então associar o valor $n$. Posteriormente, os valores devem ser normalizados para que a soma seja igual a 1 ;

b) Taxação simples - o agente de decisão deve valorar cada peso, utilizando uma escala de medida previamente escolhida (0 a 5,0 a 10,0 a 100, por exemplo). Posteriormente, os valores devem ser normalizados. 


\subsection{Tomada de decisão e modelos de otimização}

Ao longo da última década, milhões de executivos descobriram que uma das maneiras mais eficazes de analisar e avaliar alternativas de decisão envolve o uso de planilhas eletrônicas para criar modelos em computador dos problemas de decisão enfrentados. Um modelo em computador é um conjunto de relacionamentos matemáticos e suposições lógicas implementadas em um computador como representacão de algum problema ou fenômeno de decisão do mundo real.

Hoje, as planilhas eletrônicas representam a maneira mais útil e conveniente para os executivos implementarem e analisarem modelos em computador. Com o uso de um modelo de planilha (um modelo em computador implementado por meio de planilha), qualquer pessoa pode analisar alternativas de decisão antes de ter que escolher um plano específico para implementação.

Os Modelos de Otimização são utilizados quando existe o interesse em encontrar a alternativa que melhor atenda a um objetivo predeterminado. Esses modelos são formulados com a finalidade de determinar valores para um conjunto de variáveis de decisão que irão maximizar ou minimizar uma função objetivo sujeita a restrições.

Tanto a função objetivo quanto as restrições são representadas por expressões matemáticas que dependem das variáveis de decisão (WURBS, 1993). Os termos variáveis de decisão, função objetivo e restrições são frequentemente utilizados nos modelos de otimização. Ignizio (1982) define esses termos como:

_ variáveis de decisão: são variáveis que estão sob o controle do decisor e que tem influência na solução do problema de otimização;

_ função objetivo: é uma função matemática das variáveis de decisão que representa os desejos do decisor como, por exemplo, maximizar os benefícios ou minimizar os custos;

_ restrições: representam as limitações dos recursos disponíveis ou exigências específicas sobre as variáveis.

Por restrições computacionais estes modelos são usualmente obrigados a simplificar o sistema físico tratado e costumam levar desvantagem em relação à precisão, flexibilidade e representatividade dos modelos de simulação (ROBERTO e PORTO, 1999). Segundo LABADIE (1998), o maior desafio dos modelos de otimização em relação aos de simulação está na necessidade que os modelos de otimização apresentam de possuir algum tipo de modelo de simulação dentro da otimização de modo a manter a viabilidade das políticas operacionais.

Independentemente do modelo de otimização escolhido, a função objetivo é a principal parte do mesmo. Os objetivos utilizados na montagem de funções objetivos citados por WURBS (1993) dividem-se em três grupos: (1) benefícios econômicos e custos; (2) disponibilidade e confiabilidade hídrica e (3) geração de energia elétrica. Os modelos de otimização normalmente utilizam funções objetivo com um único objetivo, transformando os outros objetivos em restrições. 
Entretanto, existem modelos de otimização com mais do que um objetivo, os chamados modelos multiobjetivo.

O trabalho de Garneiro et al. (2008), apresenta resultados que permite avaliar positivamente a otimização de modelos em processos agroindustriais, pois, visualizaram potenciais ganhos no processo de suprimento a partir de sua revisão. Ainda, disseram que, a gestão de pessoas, máquinas, frotas e dos tempos é fundamental no contexto. A coordenação das atividades, objetivando maior sincronia entre as etapas interdependentes, foi capaz de gerar resultados financeiros bastante significativos no segmento de processamento industrial de tomates. As perdas de produto poderiam ser reduzidas de $2 \%$ para algo inferior a $1 \%$. A menor capacidade ociosa seria traduzida em um menor custo de oportunidade e aumento de receita. A melhor orientação dos colhedores seria capaz de reduzir perdas de caixas, por menores danos, bem como a necessidade de um menor número de caixas, pela melhor organização do trabalho de colheita. Para uma fábrica com um consumo de tomates de 336 mil toneladas por ano, a melhoria no suprimento de matériaprima poderia resultar em ganhos estimados em R $\$ 6$ milhões por ano.

Segundo Ahumada e Villalobos (2008), o principal objetivo do modelo é maximizar o valor líquido de multiperíodo das receitas. As variáveis num sistema agroindustrial podem incluir: a produção agrícola, política de perfuração, a área desenvolvida para irrigação, água e atribuição.

A principal contribuição do modelo é a de determinar em simultâneo a otimização discreta das despesas e de capital para ambos os padrões, período único e multi período.

\section{Modelo para planejamento agrícola}

Com base em programação matemática, um modelo visa compreender recursos, socioeconômicos e políticos dos principais objetivos do agricultor e deve seguir os passos como descrito na Figura 1.

Figura 1- Etapas da Modelagem de Processos

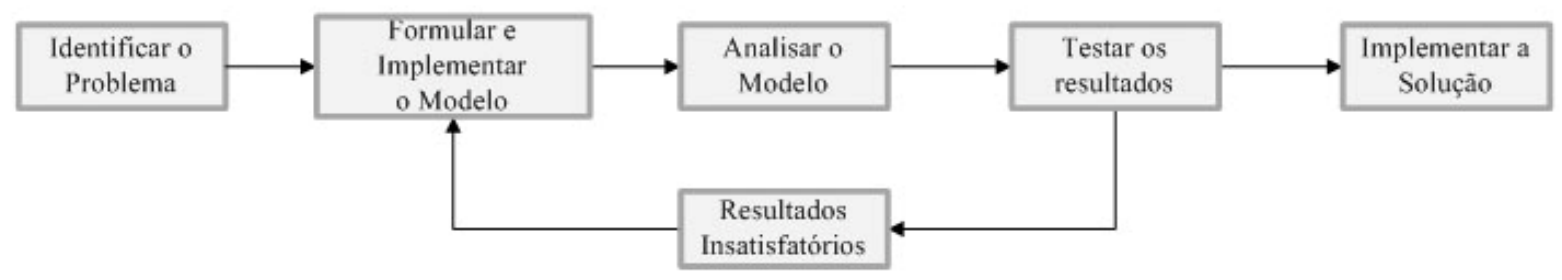

Fonte: Adaptado de Ragsdale (2009)

Segundo (Heckelei and Wolff, 2003), o uso de uma abordagem de programação matemática tem a vantagem de modelar explicitamente condições tecnológicas e políticas (obrigações, cotas de produção e restrições) em que as funções de comportamento não podem ser derivadas facilmente. 
De acordo com (Louhichi et al. 2004), os principais componentes que devem englobar a construção de um modelo para o planejamento rural são:

- Um conjunto de variáveis de decisão que descrevem as atividades agrícolas e do estado do sistema.

- Uma função objetivo que descreve o comportamento do agricultor e os objetivos em particular sobre o risco.

- Um conjunto de restrições físicas, financeiras, técnicas, econômicas e agronômicas, representando as especificações de funcionamento do sistema.

- Um conjunto de políticas e medidas ambientais (preço e mercado), cotas e obrigações, restrições à condicionalidade, etc.

\subsection{Modelos de otimização na agroindústria}

As primeiras aplicações da programação linear (PL) à economia agrícola realizaram-se no contexto da empresa agrícola (Throsby, 1974; Martin, 1977), citado por Fragoso et al (2008). Esses modelos, fáceis de construir, revelaram-se muito úteis para compreender a realidade.

A sua ampla utilização no estudo de problemas econômicos aplicados à agricultura deve-se principalmente à facilidade com que incorporam na sua estrutura os princípios da teoria econômica do produtor e ao fato das necessidades de informação serem substancialmente inferiores às dos métodos econométricos (HOWITT, 1995).

O problema econômico do produtor agrícola é geralmente formulado sob a forma primal da PL, em que o objetivo é determinar a combinação das atividades agrícolas que maximizam o lucro e que são admissíveis relativamente à disponibilidade dos recursos fixos. Frequentemente, a solução deste problema afasta-se da realidade observada e é sobre-especializada nas atividades que mais contribuem para a formação do lucro do produtor. Segundo Howitt (1995) a origem do problema de sobre-especialização da solução do modelo de PL, principalmente nos modelos agregados, está no reduzido número de restrições empíricas comparado com o número observado de atividades agrícolas na situação de referência, na falta de especificação da não linearidade das tecnologias agregadas e no fato de ser difícil considerar os preços endógenos dos produtos e o risco no comportamento dos agentes econômicos.

Como afirmam Fragoso et al (2008), no modelo de PL, as variáveis não zero, que representam as atividades agrícolas realizadas, estão limitadas pelo número das variáveis básicas do problema. Cada variável básica está associada a uma restrição do problema que, por sua vez, representa a disponibilidade dos recursos e o seu valor dual. Para cada restrição, o valor dual corresponde ao valor da produtividade marginal do recurso. Deste modo, se o número de restrições 
empíricas for inferior ao número de variáveis básicas, a solução do modelo será necessariamente sobre-especializada, verificando-se que várias atividades agrícolas observadas na situação de referência não constam da solução do modelo. Para aproximar os resultados dos modelos de PL à situação de referência, podem sempre acrescentar-se restrições que permitem aumentar o número das variáveis básicas e condicionar os valores das variáveis de decisão. Apesar da complexidade dos fatores que estão na origem da discrepância dos resultados, os esforços dos investigadores neste sentido têm sido significativos.

Como afirma Caixeta Filho (2004), uma das aplicações mais clássicas da programação linear, ramo da pesquisa operacional, diz respeito ao planejamento agrícola, ou mais genericamente, planejamento agroindustrial.

Tendo como argumento a afirmação de Caixeta Filho (2004), uma forma de responder as questões formuladas por Veloso (1997) é o emprego da pesquisa operacional, como forma de maximizar lucros e minimizar custos nos setores agroindustriais, sendo de pequeno, médio ou grande porte.

Segundo Engau (2009) muitos problemas na decisão de gestão ou financiamento, bem como várias outras áreas exigem a consideração simultânea de vários critérios e, assim, são muitas vezes modelados e resolvidos por meio de métodos multiobjetivo de programação e de tomada de decisão multicritério.

Uma característica comum a todos estes problemas é que, em geral, não existe uma única solução ideal, mas sim um conjunto dos chamados soluções de Pareto, entre os quais o decisor (DM) escolhe com base em preferências pessoais ou critérios adicionais não incluídos no modelo de otimização original.

Exemplificando, pretende-se produzir três tipos de cereais, de forma que se saiba quanto plantar ou produzir de cada um, dispondo de uma área $\mathrm{Q}$ de terreno para plantio. Seus rendimentos sejam R1, R2, R3 e seus custos de produção sejam C1, C2, C3. Deseja-se saber como maximizar a produção, obtendo o maior lucro de retorno e também como minimizar custos em função do uso de herbicidas que forem utilizados, tendo custos $\mathrm{Z} 1, \mathrm{Z} 2, \mathrm{Z3}$, obtendo a melhor área para cultivo em função dos diferentes nutrientes do solo, Figura 2. 


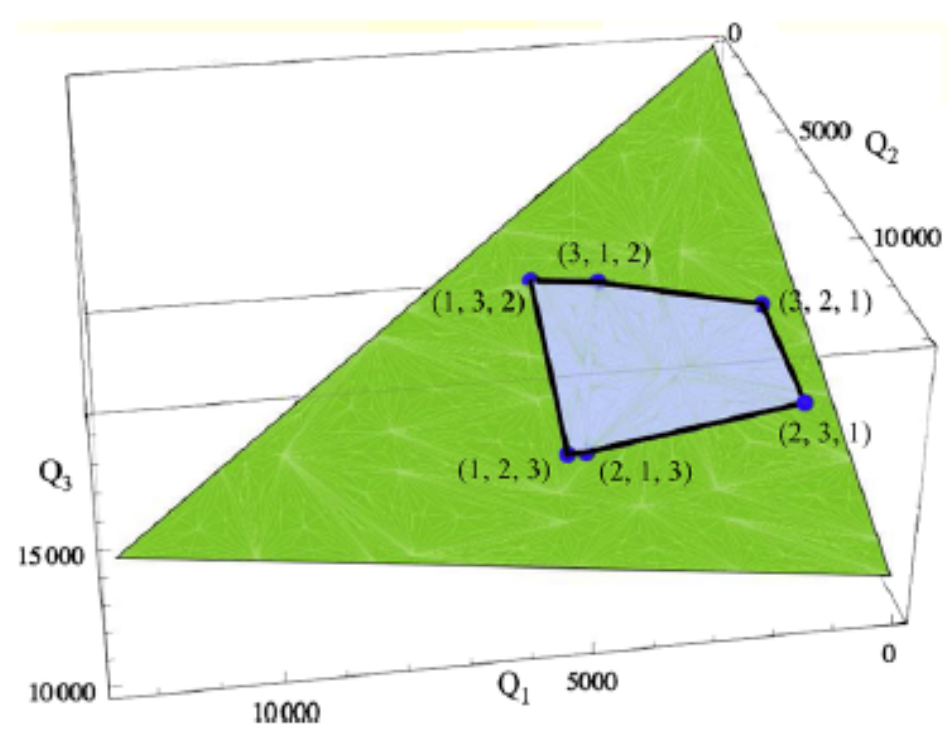

Fonte: Adaptado de Huseby e Haavardsson (2009, P. 245)

\section{Metodologia}

Para Marconi e Lakatos (2008), a pesquisa de laboratório é um procedimento de investigação mais difícil, porém mais exato. Ela descreve e analisa o que será ou ocorrerá em situações controladas. E pesquisa de campo é aquela utilizada com o objetivo de conseguir informações e/ou conhecimentos acerca de um problema, para o qual se procura uma resposta, ou de uma hipótese, que se queira comprovar, ou, ainda, descobrir novos fenômenos ou as relações entre eles.

A coleta de dados para a realização da pesquisa englobou a coleta de dados específicos do setor analisado no que diz respeito ao levantamento dos coeficientes técnicos de cada cultura, dos custos de produção para cada uma delas, consultas a acervos técnico-didáticos de periódicos on-line e literatura específica, e por fim, análise e balanceamento de operações unitárias agroindustriais.

O início do levantamento dos dados foi obtido com informações sobre o setor, em estudo, adquiridas em fontes de informação do governo brasileiro (Embrapa, SEAB) e revistas específicas sobre modelagem.

O fator chave da modelagem para a otimização de processos é relacionado com a maximização do lucro líquido das culturas selecionadas, paralelamente com a minimização de riscos de perdas e custos. Essas funções são sujeitadas as restrições que cada cultura agrícola exige e ainda pelas impostas pelo tomador de decisão.

Todas as culturas presentes na construção do modelo estarão em função de recursos aos quais necessitam para que cumpram seu ciclo e então maximizem seu rendimento. Entre eles, pode- 
se exemplificar como na tabela 1:

Tabela 1 - Restrições sujeitas ás culturas agrícolas

\begin{tabular}{c|c}
\hline \multicolumn{2}{c}{ Recursos (Restrtições) } \\
\hline Cultura & Tomador Decisão \\
\hline Sementes (Kg) & Mão-de-Obra(dh) \\
\hline Fertilizantes (Kg) & Água (L) \\
\hline Calcário (Ton) & Máquinas (hm) \\
\hline Gesso (Ton) & Energ. Elétr. (Kw) \\
\hline Herbicidas (L) & Área (ha) \\
\hline Inseticidas (L) & \\
\hline Fungicidas (L) & \\
\hline Regul. Cresc. (L) & \\
\hline Desfolhante (L) & \\
\hline Espalh. Ades. (L) & \\
\hline Fonte. O Autor &
\end{tabular}

No que se refere a ferramentas para coleta e tabulação de dados da pesquisa, o desenvolvimento do trabalho deve fazer uso de calculadoras, computadores, planilhas e formulários para modelagem matemática de processos. Entre os recursos técnicos envolvidos para a realização do estudo estariam o softwares de otimização, como o Lingo, $o$ editor de planilha eletrônica $M s$ Excel com o uso da função Solver .

Os dados da análise dos coeficientes técnicos de cada cultura são colocados em forma de planilhas, onde é possível observar o custo operacional, bem como a receita líquida de cada cultura.

Tabela 2 - Custo de Produção do Feijão

\begin{tabular}{|c|c|c|c|c|}
\hline \multicolumn{5}{|c|}{ Custo de Produção/ha da cultura do feijão tipo carioca (plantio direto) } \\
\hline Componentes & Unidade & Quantidade & Preço Unitário & Valor $(\mathrm{R} \$)$ \\
\hline A - Insumos & & & & 999,87 \\
\hline Calcário dolomítico & $\mathrm{T}$ & 1,00 & 55,00 & 55,00 \\
\hline Dessecante 1 & $\mathrm{~L}$ & 1,00 & 22,00 & 22,00 \\
\hline Dessecante 2 & $\mathrm{~L}$ & 2,00 & 34,68 & 69,36 \\
\hline Espalhante adesivo & $\mathrm{L}$ & 0,10 & 7,20 & 0,72 \\
\hline Fungicida (trat. semente) & $\mathrm{Kg}$ & 0,15 & 49,82 & 7,47 \\
\hline Adubo NPK & $\mathrm{Kg}$ & 350,00 & 0,83 & 290,50 \\
\hline Semente & $\mathrm{Kg}$ & 60,00 & 2,00 & 120,00 \\
\hline Uréia & $\mathrm{Kg}$ & 125,00 & 0,90 & 112,50 \\
\hline Herbicida PÓS & $\mathrm{L}$ & 1,00 & 135,00 & 135,00 \\
\hline Inseticida & $\mathrm{L}$ & 1,50 & 28,00 & 42,00 \\
\hline Fungicida 1 & $\mathrm{~L}$ & 0,12 & 530,00 & 63,60 \\
\hline Fungicida 2 & $\mathrm{~L}$ & 0,40 & 90,00 & 36,00 \\
\hline Fungicida 3 & $\mathrm{~L}$ & 0,40 & 107,55 & 43,02 \\
\hline
\end{tabular}




\begin{tabular}{|c|c|c|c|c|}
\hline Formicida & $\mathrm{Kg}$ & 0,30 & 9,00 & 2,70 \\
\hline B - Operações Agrícolas & & & & 324,73 \\
\hline Distribuição de calcário & $\mathrm{hm}$ & 0,20 & 35,00 & 7,00 \\
\hline Aplicação de dessecante & $\mathrm{hm}$ & 0,60 & 35,00 & 21,00 \\
\hline Tratamento de sementes & $\mathrm{dh}$ & 0,01 & 20,00 & 0,20 \\
\hline Plantio/adubação & $\mathrm{hm}$ & 0,45 & 35,00 & 15,75 \\
\hline Aplicação de uréia & $\mathrm{hm}$ & 0,25 & 35,00 & 8,75 \\
\hline Aplicação herbicida PÓS/inseticida & $\mathrm{hm}$ & 0,30 & 35,00 & 10,50 \\
\hline Aplicação de fungicida/inseticida & $\mathrm{hm}$ & 0,60 & 35,00 & 21,00 \\
\hline Aplicação de formicida & $\mathrm{dh}$ & 0,02 & 20,00 & 0,40 \\
\hline Arranquio & $\mathrm{dh}$ & 1,00 & 165,28 & 165,28 \\
\hline Trilha (recolhedora-trilhadora) & $\mathrm{hm}$ & 1,00 & 45,00 & 45,00 \\
\hline Sacaria & uma & 45,00 & 0,50 & 22,50 \\
\hline Transporte interno & $\mathrm{hm}$ & 0,21 & 35,00 & 7,35 \\
\hline C - Outros Custos & & & & 491,06 \\
\hline Análise de solo & uma & 0,50 & 20,00 & 10,00 \\
\hline Aluguel da terra & $\mathrm{sc}$ & 2,00 & 75,00 & 150,00 \\
\hline Assistência técnica & $\%$ & 1,00 & $2.625,00$ & 26,25 \\
\hline Administração & $\%$ & 2,00 & $2.625,00$ & 52,50 \\
\hline PROAGRO & $\%$ & 5,70 & $1.400,00$ & 79,80 \\
\hline Juros de custeio (6 meses) & $\%$ & 4,38 & $1.400,00$ & 61,25 \\
\hline Custo das benfeitorias & $\%$ & 1,00 & $2.625,00$ & 26,25 \\
\hline Seguro de vida & $\%$ & 0,10 & $2.625,00$ & 2,63 \\
\hline ITR & \#\#\# & 1,00 & 1,00 & 1,00 \\
\hline CESSR & $\%$ & 2,30 & $2.625,00$ & 60,38 \\
\hline Transporte externo & $\mathrm{sc}$ & 35,00 & 0,60 & 21,00 \\
\hline D - Custo Operacional & & & & $1.815,66$ \\
\hline E - Receita Bruta & & 35,00 & 75,00 & $2.625,00$ \\
\hline F - Margem operacional & & & & 809,34 \\
\hline
\end{tabular}

Fonte: O Autor

Como para cada operação e aplicação em insumos de cada cultura agrícola existe um conjunto de apontamentos de consumo de materiais e mão-de-obra, é realizado um levantamento de seus coeficientes técnicos nas bases de dados citadas acima, para que se possa obter o gasto operacional e de insumos que cada cultura demanda.

Posteriormente a essa coleta, as informações recolhidas são tabuladas no formato de uma matriz, que fornecerá os resultados das culturas relacionadas aos seus recursos, como visto na Tabela 3 .

Tabela 3: Matriz Recursos X Culturas

\section{Maximizar Lucro}

Recursos

\begin{tabular}{|c|c|}
\hline Feijão & Milho (Plantio \\
Carioca & Direto) \\
\hline
\end{tabular}

\section{Culturas}

Soja (Plantio

Direto)
Gergelim Mamona Sorgo 


\begin{tabular}{|c|c|c|c|c|c|c|}
\hline Sementes (Kg) & 60,00 & 20,00 & 81,00 & 3,00 & 6,00 & 8,00 \\
\hline Mão-de-Obra(dh) & 1,03 & 0,83 & 16,36 & 46,00 & 32,50 & 0,57 \\
\hline Água (L) & 0,00 & 0,00 & 0,00 & 0,00 & 0,00 & 0,00 \\
\hline Máquinas (hm) & 3,61 & 4,78 & 5,47 & 3,00 & 3,00 & 2,95 \\
\hline Fertilizantes (Kg) & 475,00 & 500,00 & 242,50 & 0,00 & 0,00 & 280,00 \\
\hline Calcário (Ton) & 1,00 & 0,70 & 450,00 & 0,00 & 0,00 & 0,00 \\
\hline Gesso (Ton) & 0,00 & 0,40 & 0,00 & 0,00 & 0,00 & 0,00 \\
\hline Energ. Elétr. (Kw) & 0,00 & 0,00 & 0,00 & 0,00 & 0,00 & 0,00 \\
\hline Herbicidas (L) & 4,00 & 6,30 & 5,00 & 0,00 & 0,00 & 1,50 \\
\hline Inseticidas (L) & 1,50 & 13,00 & 1,20 & 2,00 & 1,00 & 0,60 \\
\hline Fungicidas (L) & 1,07 & 0,02 & 0,62 & 0,00 & 0,00 & 0,00 \\
\hline Formicida (Kg) & 0,30 & 0,60 & 2,00 & 2,00 & 2,00 & 0,00 \\
\hline Regul. Cresc. (L) & 0,00 & 0,00 & 0,00 & 0,00 & 0,00 & 0,00 \\
\hline Desfolhante (L) & 0,00 & 0,00 & 0,00 & 0,00 & 0,00 & 0,00 \\
\hline Espalh. Ades. (L) & 0,10 & 1,00 & 0,00 & 0,00 & 0,00 & 0,00 \\
\hline Produção Kg.ha ${ }^{-1}$ & $2.100,00$ & $7.000,00$ & $3.000,00$ & 800,00 & $1.200,00$ & $2.699,00$ \\
\hline Espaço Físico Disponível (ha) & \multicolumn{6}{|c|}{40} \\
\hline Receita Bruta (R\$ . ha $\left.{ }^{-1}\right)$ & $3.600,00$ & $1.800,00$ & $2.250,00$ & $1.805,50$ & $1.200,00$ & 584,78 \\
\hline Custo Operacional(R\$ . ha $\left.{ }^{-1}\right)$ & $2.326,14$ & $1.229,86$ & $1.170,45$ & $1.040,00$ & 777,45 & 468,82 \\
\hline Receita Líquida (RS . ha ${ }^{-1}$ ) & $1.273,86$ & 570,14 & $1.079,55$ & 765,50 & 422,55 & 115,96 \\
\hline
\end{tabular}

De posse dos dados tabulados, o passo seguinte é a construção do modelo matemático baseado na pesquisa operacional em programação linear (programação multiobjetivo), ou seja, maximizar lucro e minimizar riscos.

Para BARNETT et al. (1982), a programação com múltiplos critérios apresenta a vantagem de permitir uma representação mais precisa das funções de utilidades na tomada de decisão, garantindo, assim, melhores previsões e decisões a serem tomadas.

O método de programação com múltiplos critérios pode ser caracterizado como descritivo, operacional e combinado. A abordagem descritiva é utilizada no caso em que o tomador de decisão, ao possuir múltiplos objetivos, procura hierarquizar os objetivos por meio de pesos. A abordagem operacional procura hipotetizar pesos para os objetivos, com a intenção de examinar seus impactos em um modelo de decisão. A combinação dessas abordagens procura, primeiramente, descobrir objetivos e seus pesos e, depois, utilizá-los em um modelo de decisão. 


\subsection{Análise e interpretação dos resultados}

A partir da coleta de dados e de observações das etapas em campo, pode-se se iniciar a construção dos modelos por meio de equações matemáticas determinísticas capazes de representar o encadeamento das atividades de cada cultura, sendo descrito por:

$$
\text { 1) } \begin{aligned}
\operatorname{Max}_{\mathrm{i}} & =\sum \mathrm{c}_{\mathrm{i}} \mathrm{x}_{\mathrm{i}} \\
\text { 2) } & \underset{\mathrm{i}}{\operatorname{Min}}=\sum \mathrm{d}_{\mathrm{i}} \mathrm{x}_{\mathrm{i}}
\end{aligned}
$$

onde: $c i=$ Lucro, $d i=$ Risco

sujeito a

$$
a_{1} x_{1}+a_{2} x_{2}+\ldots a_{n} x_{n}=b
$$

$$
\begin{gathered}
\mathrm{a}_{11} \mathrm{x}_{1}+\mathrm{a}_{12} \mathrm{x}_{2} \ldots \mathrm{a}_{1 \mathrm{n}} \mathrm{x}_{\mathrm{n}} \square \mathrm{b}_{1}(\text { ou } \square, \text { ou }=) \\
\mathrm{a}_{21} \mathrm{x}_{1} \mathrm{a}_{22} \mathrm{x}_{2} \ldots \mathrm{a}_{2 \mathrm{n}} \mathrm{x}_{\mathrm{n}} \mathrm{b} \square \mathrm{b}_{2}(\text { ou } \square, \text { ou }=) \\
\ldots \\
\mathrm{a}_{\mathrm{m} 1} \mathrm{x}_{1} \mathrm{a}_{\mathrm{m} 2} \mathrm{x}_{2} \ldots \mathrm{a}_{\mathrm{mn}} \mathrm{x}_{\mathrm{n}} \square \mathrm{b}_{\mathrm{m}}(\mathrm{ou} \square, \mathrm{ou}=) \\
\mathrm{x}_{1}, \mathrm{x}_{2}, \ldots, \mathrm{x}_{\mathrm{n}} \square 0
\end{gathered}
$$

onde: $x_{i}=$ Cultura,$a_{i}=$ Recursos(restrições)

\subsection{Modelagem e definicao das variaaveis de decisão}

Para a definicao das variaveis de decisao para a modelagem do planejamento do empreendimento rural e possivel estimar a tabela abaixo:

Tabela 4: Variáveis de Decisão para Planejamento Agrícola

\begin{tabular}{l|l}
\hline AGUAijk & $\begin{array}{l}\text { Quantidade de água requerida por ano pela cultura k, em metros cúbicos por hectare, para } \\
\text { cultivo do tipo j, no lote i. }\end{array}$ \\
\hline RBijk & $\begin{array}{l}\text { Receita bruta associada ao plantio de um hectare da cultura k, em reais, no lote i e sob o } \\
\text { regime de cultivo j. }\end{array}$ \\
\hline RISCijk & $\begin{array}{l}\text { Índice de risco associado ao cultivo de um hectare da cultura k, no lote i, sob o regime de } \\
\text { cultivo j. }\end{array}$ \\
\hline DAijk & $\begin{array}{l}\text { Índice de dano ambiental associado ao cultivo de um hectare da cultura k, no lote i, sob o } \\
\text { regime de cultivo j. }\end{array}$ \\
\hline MO & Disponibilidade total de mão-de-obra em dias-homem. \\
\hline MOijk & $\begin{array}{l}\text { Mão-de-obra (dias/homem), necessária à produção de um hectare da cultura k, no lote i, } \\
\text { sob o regime de cultivo j. }\end{array}$ \\
\hline ENERGIA & Disponibilidade total de energia em kwh para irrigação. \\
\hline ENERGIAijk & $\begin{array}{l}\text { Quantidade de energia requerida em kwh, para irrigação de um ha da cultura k, no lote i, } \\
\text { sob o regime de cultivo j. }\end{array}$ \\
\hline AGROTOX & Disponibilidade total de agrotóxico em kg. \\
\hline AGROTOXijk & Quantidade de agrotóxico requerida por ha da cultura k, no lote i, sob o regime de cultivo \\
\hline
\end{tabular}




\begin{tabular}{|c|c|}
\hline & $\mathrm{j}$. \\
\hline FERT & Disponibilidade total de fertilizantes, em kg. \\
\hline FERTijk & $\begin{array}{l}\text { Quantidade de fertilizantes em } \mathrm{kg} \text {, requerida por ha da cultura } \mathrm{k} \text {, no lote } \mathrm{i} \text {, sob o regime } \\
\text { de cultivo j. }\end{array}$ \\
\hline TERRAi & Disponibilidade total de terra no lote i, em hectares. \\
\hline MAQ & Disponibilidade total de equipamentos, em horas-máquina. \\
\hline MAQijk & $\begin{array}{l}\text { Quantidade de equipamento requerida, em horas-máquina, associada a um ha da cultura } \\
\mathrm{k} \text {, no lote } \mathrm{i} \text {, sob regime de cultivo } \mathrm{j} \text {. }\end{array}$ \\
\hline SEM & Disponibilidade total de sementes, em Kg. \\
\hline SEMXijk & $\begin{array}{l}\text { Quantidade de sementes requerida por ha da cultura } \mathrm{k} \text {, no lote } \mathrm{i} \text {, sob o regime de cultivo } \\
\text { j. }\end{array}$ \\
\hline CAL & Disponibilidade total de calcário, em Kg. \\
\hline CALXijk & Quantidade de calcário requerido por ha da cultura $\mathrm{k}$, no lote $\mathrm{i}$, sob o regime de cultivo $\mathrm{j}$. \\
\hline GES & Disponibilidade total de gesso. \\
\hline GESXijk & Quantidade de gesso requerido por ha da cultura k, no lote i, sob o regime de cultivo j. \\
\hline REG & Disponibilidade total de regulador de crescimento, em L. \\
\hline REGXijk & $\begin{array}{l}\text { Quantidade de regulador de crescimento requerido por ha da cultura } \mathrm{k} \text {, no lote } \mathrm{i} \text {, sob o } \\
\text { regime de cultivo } \mathrm{j} \text {. }\end{array}$ \\
\hline DES & Disponibilidade total de desfolhante, em L. \\
\hline DESXijk & $\begin{array}{l}\text { Quantidade de desfolhante requerido por ha da cultura k, no lote i, sob o regime de } \\
\text { cultivo j. }\end{array}$ \\
\hline ESP & Disponibilidade total de espalhante adesivo em L. \\
\hline ESPXijk & $\begin{array}{l}\text { Quantidade de espalhante adesivo requerido por ha da cultura k, no lote i, sob o regime } \\
\text { de cultivo j. }\end{array}$ \\
\hline
\end{tabular}

Fonte: O Autor

Após a construção dos modelos, os mesmos são implementados em computador, através dos programas Excel ou Lingo.

Posteriormente, esses programas fazem a resolução matemática dos problemas, oferencendo a solução ótima de cada cultura, ou seja, fornecendo nos resultados, valores ótimos de produção.

Ainda, através das resoluções via software, esses programas fornecem o valor ótimo global da função maximizada a que foi sujeitada, oferecendo assim o melhor rendimento em termos de capital disponível. Conforme Figura 3:

Figura 3: Maximização da Função Lucro (Conforme matriz tabela 3)

\begin{tabular}{|c|c|}
\hline $\begin{array}{c}\text { Max Z } \\
=\end{array}$ & $1273,86 \mathrm{X}_{1}+570,14 \mathrm{X}_{2}+1079,55 \mathrm{X}_{3}+765,50 \mathrm{X}_{4}+422,55 \mathrm{X}_{5}+115,96 \mathrm{X}_{6}$ \\
\hline
\end{tabular}

Os resultados obtidos a partir do modelo e com os parâmetros de ponto ótimo gerados pelo programa são comparados com informações empíricas levantadas em campo.

Assim, que estas são validadas, podem fornecer ao tomador de decisão a melhor estratégia de produção a ser adotada para determinado período, ou seja, quais culturas agrícolas devem ser 
plantadas e mantidas de modo que o lucro seja máximo e sejam respeitadas as restrições de seus recursos (solo, mercado comprador, dos equipamentos disponíveis, área, etc).

Consequentemente, pode-se definir quanto de área da propriedade do tomador de decisão será destinada a cada cultura, formando um esquema de distribuição como indicado na Figura 4:

Figura 4: Distribuição de Culturas após processo de otimização

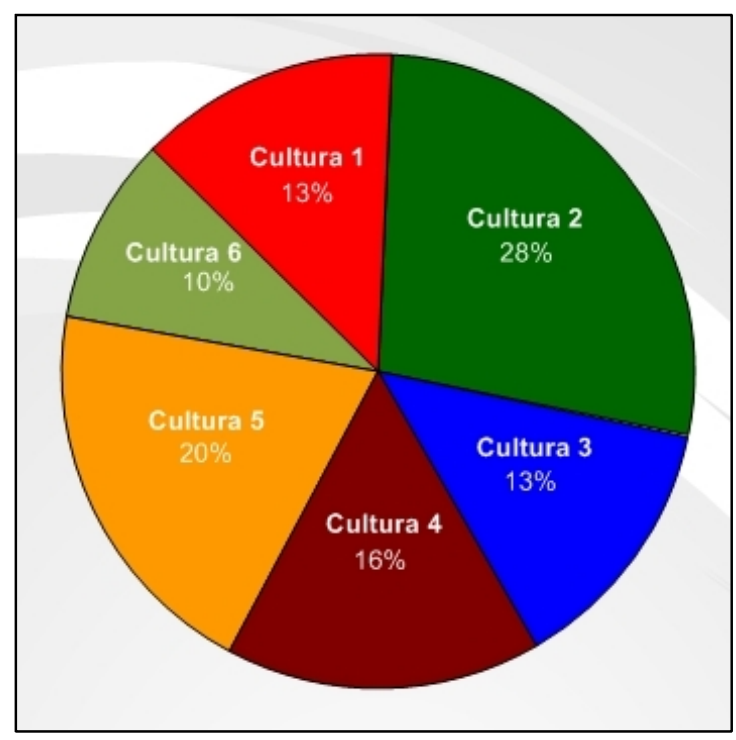

Fonte: O Autor

Para melhorar a definição dos procedimentos que objetivam a modelagem dos processos de otimização seguidos neste trabalho, seguem os seguintes passos:

a) Maximiza-se a renda líquida sujeita às restrições, com o emprego do Módulo Solver do Ms Excel, ou com o uso do software otimizador Lingo já referido anteriormente;

b) Desde que a solução encontrada na etapa anterior não seja adequada para os objetivos do tomador de decisão, identifica-se qual recurso produtivo é o mais limitante. Corresponde ao de menor relação Restrição de Recurso / Coeficiente Técnico da variável de decisão de maior renda líquida / hectare, em valores absolutos. Tal relação representa a área máxima a ser plantada com cada cultura, com o uso total do respectivo recurso nesta cultura;

c) Identificado o recurso mais limitante e desde que seja viável obter aporte adicional do mesmo, faz-se uma análise de sensibilidade das soluções pontuais, em função de acréscimos constantes à disponibilidade do recurso mais limitante. Ou seja, haverá uma solução produzida pelo Solver, para cada nível desse recurso;

d) Elabora-se uma planilha demonstrativa dos resultados obtidos com a solução, que entre as obtidas na análise de sensibilidade, mais se aproximar das pretensões do tomador de decisão; 
Passa-se então ao outro objetivo desejado, o de minimizar os riscos de perda, ou riscos econômicos das culturas, onde se procurará hierarquizar os objetivos por meio de pesos.

A abordagem operacional será então a de hipotetizar os pesos para os objetivos, com a intenção de examinar seus impactos no modelo de decisão.

Esta atribuição de pesos será levada em consideração com a porcentagem do risco, ou ainda, com a probabilidade do risco a que a determinada cultura esteja ligada.

Uma forma de demonstrar esses riscos é ilustrada na Figura 5:

Figura 5 - Riscos sofridos por Culturas Agrícolas

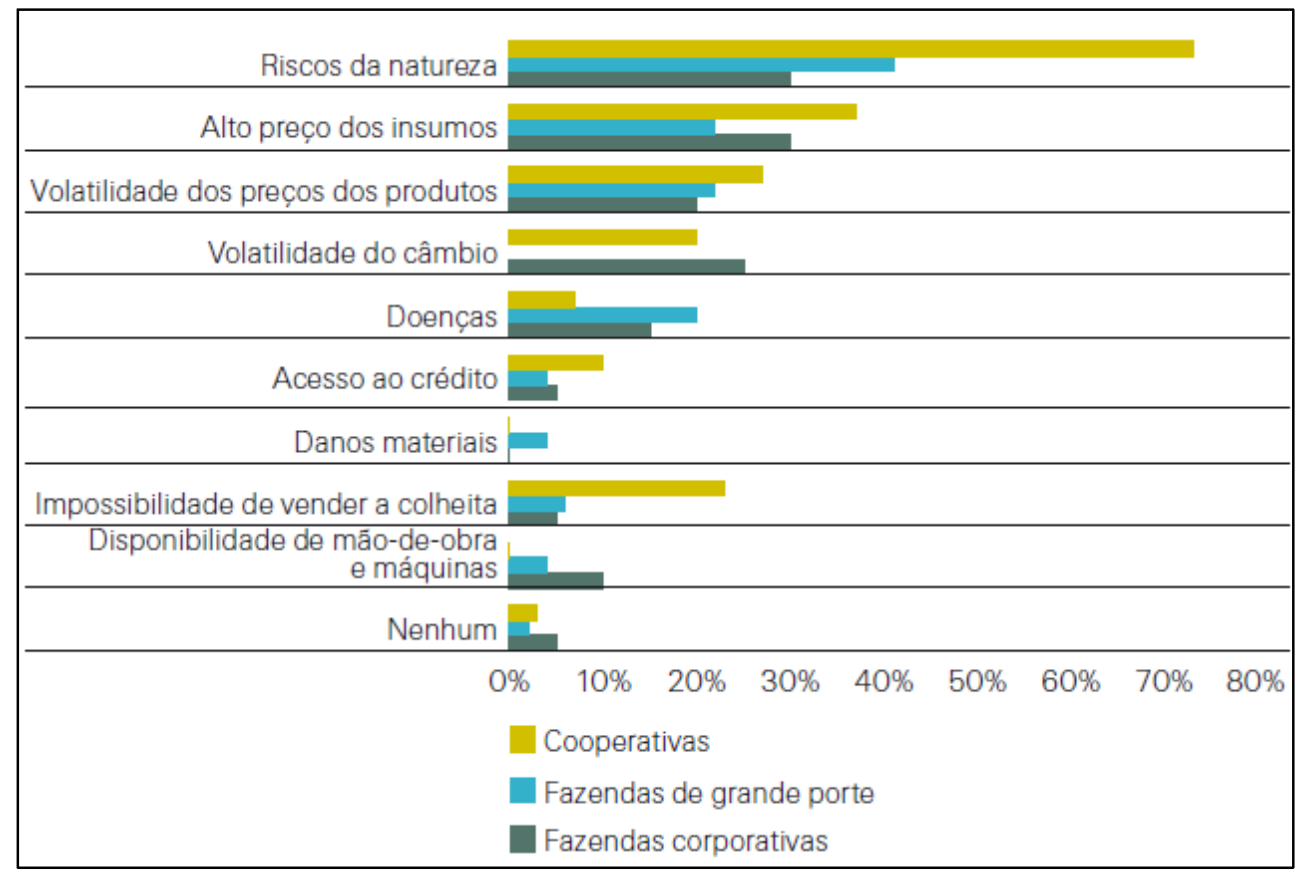

Fonte: O Autor

\section{Conclusão}

Em geral, pessoas ligadas ao planejamento agrícola normalmente não tem acesso a novas tecnologias para a aplicação em seus projetos e ações, seja pelo poder aquisitivo mais baixo ou até mesmo pelo hábito de cultivar velhas práticas. Contudo, é comum o desejo de implantar algum processo de melhoria que apresente vantagem competitiva frente a seus concorrentes.

A inserção de modelos de otimização no planejamento agrícola requer a confecção de modelos matemáticos que na maioria das vezes podem ser complexos se realizados com muitas variáveis.

Como forma de prever custos e gastos desnecessários, o emprego de modelos de decisão pode funcionar eficazmente, apresentando alto equilíbrio quando modelado em regime multicriterial. 
Os modelos construídos com atribuição de pesos tornam os modelos mais próximos da realidade e facilitam a validação do modelo, porém é necessário que ocorra uma análise criteriosa quando da efetivação desses pesos.

O estudo teórico dos parâmetros empregados na construção dos modelos de otimização neste artigo mostra que o processo da coleta de dados e a tabulação de coeficientes técnicos das culturas constituem a parte mais importante do planejamento agrícola. No entanto, o detalhamento na formulação das variáveis de decisão se torna crucial para uma produção mais precisa e com menos possibilidade de erros.

Por fim, espera-se com a construção destes modelos, que haja um melhor aproveitamento dos processos que envolvem a agroindústria, seja ela de pequeno, médio ou grande porte, mas em especial, que favoreça os pequenos produtores que, já por conseqüência de mercado e questões financeiras, são de longe os mais atingidos pelas perdas em processos de fabricação, colheita, plantio, logísticas de entregas ou pela simples economia na hora de produzir.

\begin{abstract}
The agroindustrial sectors are increasingly enhancing the technological knowledge within their fields, not only by competition, which is increasingly fierce, but also by the very simple and survival in the market. This means that there is a need to achieve an optimization of actions, which in the case of the agribusiness is translated as producing and growing food-saving investments, avoiding the waste of raw materials, inputs and unnecessary spending even more because to be an industry that relies on the adversities of weather. Operations research as a science, with structure processes, proposing a set of alternative actions, making the prediction and comparison of values, efficiency and cost. Already the optimization models, are used when there is an interest in finding the alternative that best meets one or several goals simultaneously, given a set of constraints, usually linear. Taking the principle of obtaining optimal solutions to agro-industrial processes of planning and choice of crops, the aim of this study was to determine parameters to be used to build any optimization models and should be considered so that there is the maximization profits on farms with better use of land, capital and manpower in production systems proposed, as well as minimizing the risks of opting for certain crops.
\end{abstract}

Keywords: optimization, agribusiness, operations research crops.

\title{
Referências
}

AHUMADA, O VILLALOBOS, R Application of planning models in the agri-food supply chain: A review. European Journal of Operational Research 195 (2009) 1-20. Department of Industrial Engineering, Arizona State University.

CAIXETA-FILHO, J.V. Pesquisa Operacional: Técnicas de Otimização aplicadas a Sistemas Agroindustriais. 2. Ed. São Paulo: Editora Atlas S.A, 2004.

ENGAU, A; M.M. Wiecek, Introducing nonpolyhedral cones into multiobjective programming, in: V. Barichard, M. Ehrgott, X. Gandibleux, V. T'kindt (Eds.), Multiobjective Programming and Goal Programming: Theoretical 
Results and Practical Applications, Lecture Notes in Economics and Mathematical Systems, vol. 618, Springer, Berlin, 2009, pp. 35-45 (Chapter 4).

FERRER, J.C., MACCAWLEY, A., MATURANA, S., TOLOZA, S., VERA, J., 2008. An optimization approach for scheduling wine grape harvest operations. International Journal of Production Economics 112 (2), 985-999.

cross ${ }^{\text {ref }}$

FRAGOSO RUI, M. S; CARVALHO, MARIA. L.S; HENRIQUES, PEDRO D. S. A Programação Matemática Positiva como Instrumento de Calibração e Prescrição dos Modelos de Oferta Agrícola. Investigação Operacional, Évora, v.28, p.1-16, 2008.

GAMEIRO, Augusto Hauber; CAIXETA-FILHO, José Vicente; ROCCO, Cleber Damião;

RANGEL, Rogério. Modelagem e gestão das perdas no suprimento de tomates para processamento industrial. Revista Gestão e Proução, São Carlos, v. 15, n. 1, p. 101-115, jan.-abr. 2008

GOMES, L.F.A.M.; GOMES, C.F.S.; ALMEIDA, A.T. Tomada de Decisão Gerencial: Enfoque Multicritério. São Paulo: Atlas, 2002.

HAMER, P.J.C., 1994. A decision support system for the provision of planting plans for Brussels sprouts. Computers and Electronics in Agriculture 11, 97-115.

HAZELL, P.B.R., NORTON, R.D., 1986. Mathematical Programming for Economic Analysis in Agriculture. Macmillan Publishing Company, New York, NY.

HECKELEI,T.; WOLFF,H. Estimation of constrained optimisation models for agricultural supply analysis based on generalized maximum entropy. European Review of Agricultural Economics, p.27-50, 2003.

HECKELEI, T. AND BRITZ, W. (2005) Models Based on Positive Mathematical Programming: State of the Art and Further Extensions, In Arfini, F. (Ed.): Modelling Agricultural Policies: State of the Art and New Challenges, Parma, Italy, pp. 48-73.

HIGGINS, A., THORBURN, P., ARCHER, A., JAKKU, E., 2007. Opportunities for value chain research in sugar industries. Agricultural Systems 94, 611-621.

HOFFMANN, R . et al. Inovações tecnológicas e transformações recentes na agricultura brasileira. Relatório de Pesquisa, Piracicaba: FEALQ, 1985, 4v, 480p.

HOWITT, R. (1995) Positive Mathematical Programming. American Journal of Agricultural Economics, 77 (2), pp. 329-342.

IGNIZIO, J. P., Linear Programming in Single \& Multiple-Objective Systems, Prentice-Hall, 1982.

JUNQUEIRA, Rogério Ribeiro; MORABITO, Reinaldo. Um modelo de otimização linear para o planejamento agregado da produção e logística de sementes de milho. Revista Produção, v. 16, n. 3, p. 510-525, Set./Dez. 2006.

LABADIE, J. W., Reservoir System Optimization Models. In: Decision Support Systems Applied to Water Resources Engineering, São Paulo, 1998.

LACHTERMACHER, G . Pesquisa Operacional na Tomada de Decisões. 4 ed. São Paulo: Prentice Hall, 225 p. 2009.

LAZZARI, M., MAZZETTO, F., 1996. A PC model for selecting multicropping farm machinery system. Computers and Electronics in Agriculture 14, 43-59.

LOESCH, C. \& HEIN, N. Pesquisa Operacional - Fundamentos e Modelos. São Paulo: Saraiva, 2009.

MAATMAN, A., SCHWEIGMAN, C., RUIJS, A., VAN DER VLERK, M.H., 2002. Modeling farmer's response to uncertain rain fall in Burkina Faso: A stochastic programming approach. Operations Research 50 (3), $399-414$. cross ${ }^{\text {ref }}$

MARCONI, M. A.; LAKATOS, E. M. Pesquisa bibliográfica. In: Fundamentos da Metodologia científica. 6. ed. São Paulo: Atlas, 2001. p. 43-77. 
MARTIN, L.R. (1977) A Survey of Agricultural Economics Literature. vol 2, University of Minnesota Press, Minneapolis.

MCCARL, B.A., NUTHALL, P., 1982. Linear-programming for repeated use in the analysis of agricultural systems. Agricultural Systems 8 (1), 17-39.

cross ${ }^{\text {ref }}$

MEISTER, A., CHEN,C. AND HEADY, E. (1978) Quadratic Programming Models Applied to Agricultural Policies, Iowa State University Press, Ames.

NEVO, A., OAD, R., PODMORE, T., 1994. An integrated expert system for optimal crop planning. Agricultural Systems 45, 73-92.

cross ${ }^{\text {ref }}$

PEROSIO, D.J., MCLAUGHLIN, E.W., CUELLAR, S., PARK, K., 2001. Supply chain management in the produce industry. Produce Marketing Association, Newark, Delaware, 22-32.

PINHEIRO, J.C.V.; COELHO, M.E.H \& AGUIAR, J.V. Planejamento Multicriterial para Fruticultura: O Caso do Vale do Trussu em Iguatu - CE. Revista Teoria e Evidência Econômica, Vol.13, n.24, p. 9-23, 2005.

PORTO, R. L. et al., Sistema de Suporte a Decisões para Operação dos Grandes Sistemas Produtores da SABESP, Décimo Terceiro Simpósio Brasileiro de Recursos Hídricos, Belo Horizonte, ABRH, 1999.

PRADO, D.S, Programação Linear, Nova Lima, MG. 238p. 2004.

RAGSDALE, Cliff T. Modelagem e Análise de Decisão. São Paulo: Cengage Learning, 2009.

RANTALA, J., 2004. Optimizing the supply chain strategy of a multi-unit finish nursery. Silva Fennica 38 (2), 203-215.

RECIO, B., RUBIO, F., CRIADO, J.A., 2003. A decision support system for farm planning using AgriSupport II. Decision Support Systems 36 (2), 189-203.

cross'

SANDERS, N R; (1995) Managing the forecasting function. Industrial Management \& Data Systems, MCB University Press, v. 95, n.4, p.12-18.

THROSBY, C. (1974) New methodologies in agricultural production economics: A review. In The Future of Agriculture. Papers and reports, 15 th international Conference of Agricultural Economics, S. Paulo, Brazil, pp. 150169.

TUNG, N. H. Planejamento e controle financeiro das empresas agropecuárias. São Paulo: Edições UniversidadeEmpresa, 1990. 382 p.

TURVEY, C.G., BAKER, T.G., 1990. A farm-level financial analysis of farmer's use of futures and options under alternative farm programs. American Journal of Agricultural Economics, 946-957.

VELOSO, R.F. Planejamento e Gerência de Fazenda: Princípios Básicos para Avaliação de Sistemas Agrossilvipastoris nos Cerrados. Cadernos de Ciência \& Tecnologia, Brasília, v.14, v.1, p.155-177, 1997.

VILCKAS, M. Determinantes da tomada de decisão sobre as atividades produtivas rurais: proposta de um modelo para a produção familiar. 2004. 143f. Dissertação (Mestrado em Engenharia de Produção) - Universidade Federal de São Carlos, São Carlos.

WURBS, R., Reservoir-System Simulation and Optimization Models, Journal of Water Resources Planning and Management, ASCE, v.119, n.4, p.455-472, 1993.

cross ${ }^{\text {ref }}$

ZUIN, L.F.S; QUEIROZ, T.R. Agronegócios: Gestão e Inovação. São Paulo: Saraiva, 2006. 


\section{Dados dos autores:}

Nome completo: Carlos Alessandro Neiverth Oliszeski

Filiação institucional: UTFPR - Ponta Grossa

Departamento: PPGEP

Função ou cargo ocupado: Aluno de Mestrado de Engenharia de Produção

Endereço completo para correspondência: Rua Coronel Dulcidio 1839, Ponta Grossa Paraná/Brasil.

CEP: 84010-280

Telefones para contato: 42 3224-5468 / 42 8812-0604

e-mail:carlosoliszeski@uol.com.br

Nome completo: João Carlos Colmenero

Filiação institucional: UTFPR - Ponta Grossa

Departamento: PPGEP

Função ou cargo ocupado: Professor pesquisador

Endereço completo para correspondência: Av Monteiro Lobato, s/n - Km 04

Telefones para contato: $3220-4826$

e-mail: colmenero@utfpr.edu.br 